\title{
The effect of Attentional Focus Conditions on Performer's EMG Activity
}

\author{
${ }^{* 1}$ Ayse Nur Ay, ${ }^{2}$ Baris Dolukan and ${ }^{3}$ Mustafa Zahid Yildiz \\ ${ }^{* 1}$ Faculty of Technology, Department of Mechatronics Engineering Sakarya University, Turkey \\ ${ }^{2}$ Institute of Natural Science, Biomedical Engineering Department, Sakarya University, Turkey \\ ${ }^{3}$ Faculty of Technology, Electric and Electronics Engineering Department, Sakarya University, Turkey
}

\begin{abstract}
Many researchers have been investigated the effect of focusing type on motor performance and muscular activity. Studies have showed that focusing on movement effects, i.e., an external focus enhances motor performance whereas, reduces muscular activity compare to focusing on body movements, i.e., an internal focus. The purpose of present study was to determine whether focusing externally had any effect on reduction of Electromyographic activity of performers. In this study, 16 participants were asked to perform weight lifting under control (no instructions are given), external (focusing on dumbbell) and internal (focusing on biceps brachii muscle) conditions. The EMG data were acquired via DELSYS EMG System and monitored on LabVIEW. RMS and RMSE values were calculated to compare the EMG activities under each conditions. The results showed that the EMG activity was significantly reduced under external focus condition.
\end{abstract}

Key words: External focus, Internal focus, EMG activity

\section{Introduction}

In order to facilitate skill learning and optimize the performance of the people especially athletes and sportsmen, the performer's focus of attention has interested by both researches and practitioners as a potentially significant factor for many years. Early studies were demonstrated that the focus of attention induced by the instructions or feedback provided to learners can have a significant impact on motor skill learning $[1,2,3]$. Over the past 20 years attentional focus differences have been found to have an essential influence on the speed of learning process and effectiveness and efficiency of motor performance. The first study which establishes the differences of external (focusing on the movement effect) versus internal focus (focusing on body movements) on learning was published by Wulf, Höß, \& Prinz in 1998 and then many studies have followed them [4]. Most studies on attentional focus have used measures of either effectiveness (e.g., accuracy, balance) or efficiency (e.g.,Electromyography, heart rate and force production). In fact, some have examined both aspects [5]. There are many studies in which the external focus enhance motor performance and learning skills and also reduce EMG activity of performers [6]. In order to examine muscular activity under different attentional focus conditions, biceps curl tasks have performed in many studies. The first study that examine the muscular activity was published by Vance, Wulf, Töllner, McNevin, and Mercer [7]. In this, participants were asked to focus on the weight bar (external focus) and then to their arms (internal focus). Integrated EMG activity (iEMG) in both biceps and triceps brachii muscles was measured. The outcomes showed that there was a

*Corresponding author: Address: Faculty of Technology, Department of Mechatronics Engineering Sakarya University, Sakarya TURKEY. E-mail address: ay@sakarya.edu.tr, Phone: +902642956493 
significant difference between external and internal focus condition's results and the iEMG value was higher when participants were focused externally.

This study is based on determining muscular activity changes under control, internal and external focus conditions.

\section{Materials and Method}

In order to obtain the differences of EMG activity results under various attentional focus conditions, how to measure the EMG activity is the key point. To facilitate measuring EMG activity of people, it is important to know which muscle will be played an active role to make the movement effective. Not only the muscles, but also the purpose of the experimental task must be clarified as well.

\subsection{Design of Experimental Tasks}

There are several ways to design a task for measuring muscle activities under different attentional focus conditions. In this study, some tasks that are found in the literature were used as reference. For example, in one of the recent study, the effect of attentional strategies on children performance and their EMG activities in maximum a force production has been published [8]. In this, 20 children were participated to the experiments and they were not aware of specific purpose of the study. The task was simply based on performing vertical jumps in order to reach the highest rung on Vertec. EMG activity measurement was taken from 5 different muscles and height of their jumps were measured under three type of attentional focus conditions as well. RMSE values were used to obtain muscular activity. The jump height results are shown in Figure 1-a and the EMG RMSE results are displayed in Figure 1-b.
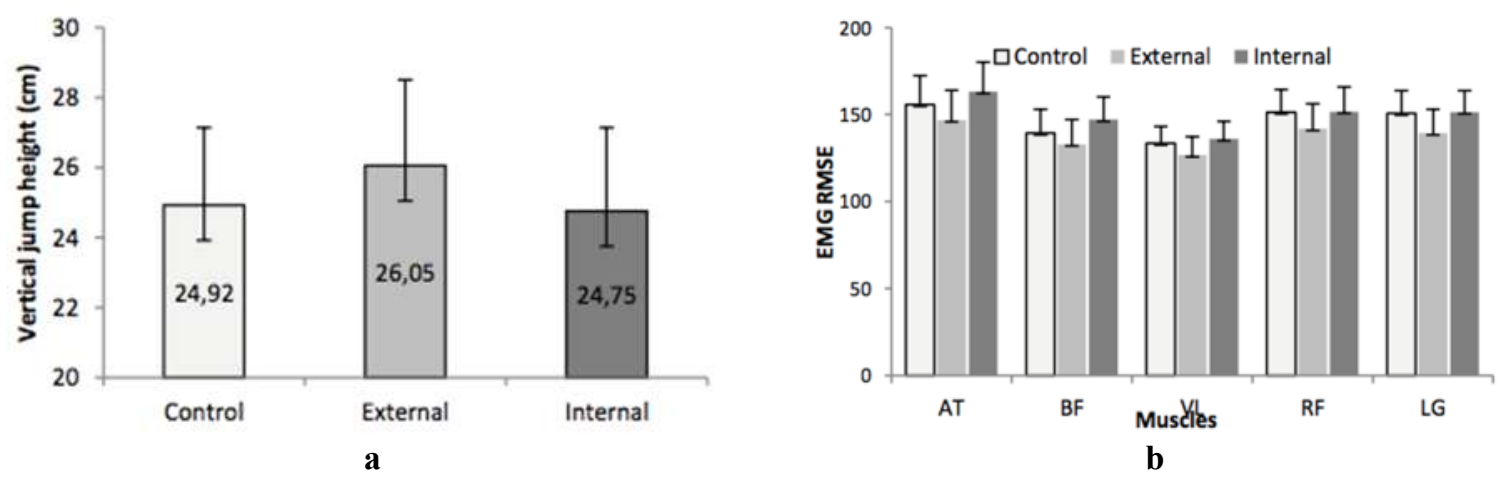

Figure 1-a: Jump and Reach height as a function of internal versus external focus

Figure 1-b:EMG RMSE from muscle onset to takeoff, for the various muscles

These results show that children in the force production tasks would better perform jumping when adopting external focus rather than adopting the internal one. Based on these basic tasks, the preliminary experiment for this study has been designed. 


\subsubsection{Preliminary Experiments}

The purpose of the study was determining the optimum methods for the muscular activity measurements from specific muscles and developing possible algorithms to analyze the EMG signals. 16 participants ( 8 female, 8 male, average age $=22$ ) was involved to the experiments. A 2 $\mathrm{kg}$ dumbbell was used. Participants were asked to lift the dumbbell with their dominant hands while sitting on a chair. The procedure was simply doing up and down exercises and when the dumbbell was up, contracting the biceps brachii muscle for 5 seconds. The procedure was repeated three times and each trial was performed under control (no instructions are given), external (focusing on the dumbbell) and internal (focusing on the biceps brachii muscle) focus conditions. The EMG signals were measured using Delsys EMG system and the data was acquired using NI LabVIEW and its biomedical toolkit (Figure 2). None of the participants were aware of the purpose of the study.

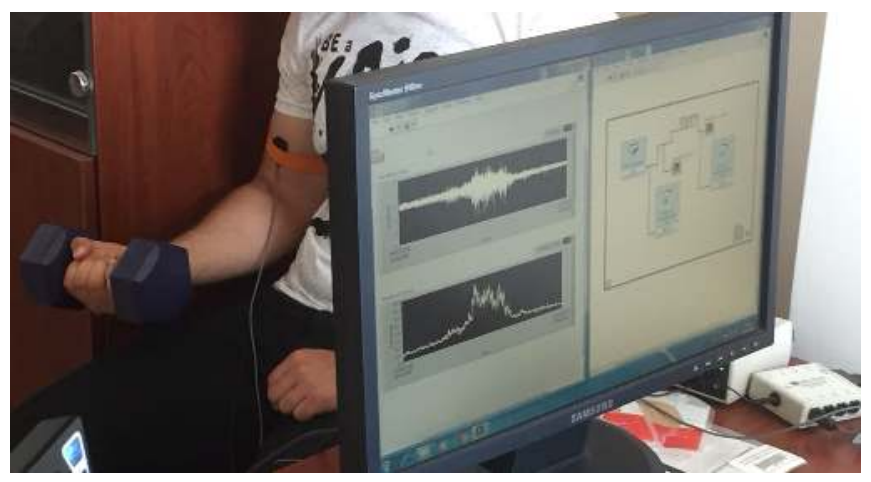

Figure 2: Experimental Procedure

After acquiring of EMG data from Delsys, an algorithm was developed in LabVIEW for preprocessing the signals.

\subsubsection{Preprocessing of EMG Signals}

Once the signal is acquired in LabVIEW environment, the next step was processing the signal for feature extraction purpose. Firstly, a Butterworth band pass filter was used which is a combination of low pass and high pass filter. Since most of the EMG signal contents lie in this region, here lower cutoff frequency was set to be $20 \mathrm{~Hz}$ whereas, higher cutoff frequency was set to be $500 \mathrm{~Hz}$ [9]. The low pass filter was used for removing environmental noise and the high pass filter was used to attenuate DC offset noise voltage.

\subsubsection{Root Mean Square (RMS) and RMS Error Calculation}

The Root Mean Square (RMS) is a statistical measure of magnitude of varying quantity. The RMS value of a continuous time waveform is the square root of arithmetic mean of the squares of the original values [10]. The RMS value calculation is given in formula 1. 


$$
\begin{gathered}
\text { Vrms }=\sqrt{\frac{1}{N} \sum_{n=1}^{N} x_{n}^{2}} \\
\begin{array}{c}
\mathrm{V}_{\mathrm{rms}}=\text { the root mea square value } \\
\mathrm{Xn}=\text { value of samples } \\
\mathrm{n}=\text { number of samples }
\end{array}
\end{gathered}
$$

A block diagram for calculating RMS value in LabVIEW environment is given in Figure 3.

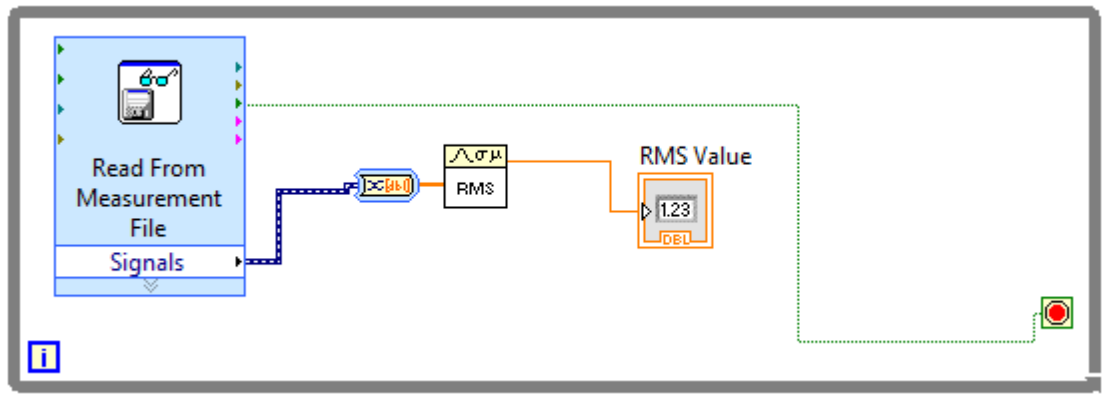

Figure 3: RMS Function in LabVIEW

The RMS values were calculated for each participants under three different attentional focus conditions and the results were tabled. Based on several studies, RMS error values were calculated out of RMS values $[11,12,13]$. As a next step, RMS Error values were calculated between control and external focus and between control and internal focus conditions. The calculation of RMSE value is given in formula 2 [14]. Two simple sample of calculation of RMSE values for the experimental results are given in Table 1.

$$
\begin{aligned}
& \boldsymbol{R M S E}= \sqrt{\frac{\sum_{i=1}^{n}(\boldsymbol{p i}-\boldsymbol{a i})^{2}}{n}} \\
& \mathrm{a}=\text { actual target } \\
& \mathrm{p}=\text { predicted target }
\end{aligned}
$$

Table 1. RMSE Calculation

\begin{tabular}{ccccc} 
EXTERNAL & CONTROL & RESIDUALS & SQUARED & RMSE \\
\hline 0.0111 & 0.016 & -0.0049 & 0.00002401 & 0.004964751 \\
& & & & \\
INTERNAL & CONTROL & RESIDUALS & SQUARED & RMSE \\
0.0089 & 0.016 & -0.0071 & 0.00005041 & 0.021191935
\end{tabular}




\section{Results}

In order to obtain the differences between attentional focus conditions groups, firstly RMS values were analyzed. Paired t-test $(\mathrm{p}=0.05)$ was applied to the data and the results are given in Table 2.

Table 2. Statistical Results

t-Test: Paired Two Sample for Means

\begin{tabular}{lrr}
\hline & EXTERNAL & INTERNAL \\
\hline Mean & 0,097675 & 0,11360625 \\
Variance & 0,005832063 & 0,007896385 \\
Observations & 16 & 16 \\
Pearson Correlation & 0,948482135 & \\
Hypothesized Mean & & \\
Difference & 0 & \\
Df & 15 & \\
& - & \\
t Stat & 2,178953665 & \\
$\mathrm{P}(\mathrm{T}<=\mathrm{t})$ one-tail & 0,022845982 & \\
t Critical one-tail & 1,753050356 & \\
$\mathrm{P}(\mathrm{T}<=\mathrm{t})$ two-tail & 0,045691965 & \\
$\mathrm{t}$ Critical two-tail & 2,131449546 & \\
\hline
\end{tabular}

The paired t-test results showed that the $\mathrm{p}$ value was less than 0,05 which means there was a significant difference between external and internal focus conditions in their muscular activity measurement.

Secondly, RMS Error values were calculated (control-external vs. control-internal) for each participant and paired t-test was applied to the results. However, there was no significant differences between external focus and internal focus conditions.

\section{Discussion}

The main purpose of the present study was to examine the advantage of external focus on muscular activity in adults' performance. There are many studies which have proved that the EMG activity is decreased in external focus $[15,16,17]$. Apart from these studies a control condition was implemented to this study. Consistent with many results it was found greater EMG activity in the internal compared to the external focus condition in adults' performance by comparing their RMS results. However, RMSE values have not given any significant results. Since control condition was individual for each performers, without given any instructions, one cannot determined reliable results. Even though RMSE values seem to be different from each other, number of performers were not found enough to conclude any statistical results. By increasing participants' number, it is 
highly possible to obtain differences statistically as well. These findings have showed that adults in such as force production, grasping or weight lifting tasks would better perform with less muscular activity when adopting external focus rather than adopting the internal one. Based on these findings it can be determined that an external focus of attention compared to the internal focus have an advantage in muscular activity in adults' performance. In this study, when participants were instructed to focus on their biceps brachii muscles (internal focus) instead of focus on dumbbells (external focus), the EMG activity was found to be greater. These findings are in line with constrained action hypothesis [18].

\section{Conclusions}

In this study, it has been investigated that whether focusing externally had any effect on reduction of EMG activity of performers. For that, 16 participants were asked to perform weight lifting under control (no instructions are given), external (focusing on dumbbell) and internal (focusing on biceps brachii muscle) conditions. The EMG data were acquired via DELSYS EMG System, monitored on processed on LabVIEW. RMS and RMSE values were calculated to compare the EMG activities under each conditions. The RMS results showed that the EMG activity was significantly reduced when the performers focused externally.

\section{References}

[1] C. Shea and G. Wulf, "Enhancing motor learning through external focus instructions and feedback," Human Movement Science, vol. 18, pp. 553-571, 1999.

[2] G. Wulf, B. Lauterbach and Toole T., "Learning advantages of an external focus of attention in golf," Research Quarterly for Exercise and Sport, vol. 70, pp. 120-126, 1999.

[3] G. Wulf, N. McNevin and T. Fuchs, "Attentional Focus in complex motor skill learning.," Research Quarterly for Exercise and Sport, vol. 71, pp. 229-239, 2000.

[4] G. Wulf, M. Ho" ß and W. Prinz, "Instructions for motor learning: Differential effects of internal versus external focus of attention," Journal of Motor Behavior, vol. 30, pp. 169-179, 1998.

[5] G. Wulf, "Attentional Focus and Motor Learning: A review of 15 years," International Review of Sport and Exercise Psychology, vol. 6, pp. 77-104, 2013.

[6] D. Marchant, M. Greig and C. Scott, "Attentional focusing strategies influencebicep EMG during isokinetic biceps curls," Athleti Insight, 2008. 
[7] J. Vance, G. Wulf, T. Töllner, N. McNevin and J. Mercer, "EMG Activity as a Function of the performers' focus of attention," Journal of Motor Behavior, vol. 36, pp. 450-459, 2004.

[8] A. Ramin, A. Mohammad Taghi and S. Mansor, "The effect of attentional focus strategies on chidren performance and their EMG activities in maximum a force production task," Turkish Journal of Kinesiology, 2017.

[9] Fabiano Politti, Claudia Casellato, Marcelo Martins Kalytczak, Marilia Barbosa Santos Garcia and Daniela Aparecida Biasotto-Gonzales, "Characteristics of EMG frequency bands in temporomandibullar disorders patiens," Journal of Electromyography and Kinesiology, vol. 31, pp. 119-125, 2016.

[10] Y. Singh, Analysis Bio-Potential to Ascertain Movements for Prosthetic Arm with Different Weights Using LabVIEW, India, 2013.

[11] Wulf, G, Weigelt, M, Poulter, D. R and McNevin,N. H, “Attentional focus on suprapostural tasks affects balance learning," Quarterly Journal of Experimental Psychology, vol. 56, pp. 1191-1211, 2003.

[12] Scott W.T. MvNamara, Kevin A. Becker and Lisa M., "The differential effects of attentional focus in children with moderate and profound visual impairments," Front Psychol, vol. 8, 2017.

[13] Gabriele Wulf, "Attentional Focus Effects in Balance Acrobats," Research quarterly for exercise and sport, vol. 79, pp. 19-25, 2008.

[14] "Model Evaluation - Regression," [Online]. Available: http://www.saedsayad.com/model_evaluation_r.htm.

[15] Wulf G. and Dufek JS., "Increased jump height with anexternal focus due to enhanced lower extremity joint kinetics," Journal of Motor Behavior , vol. 41, pp. 401-409, 2009.

[16] Wulf G., Zachry T., Granados C. and Dufek JS., "Increases in jump-and reach height through an external focus of attention," International Journal of Sports Science and Coaching, vol. 2, pp. 275284, 2007.

[17] Wulf G., Dufek JS, Lozano L. and Pettigrew Ch., "Increased jump height and reduced EMG activity with an external focus.," Human Movement Science, vol. 29, pp. 440-448, 2010.

[18] Wulf G., McNevin N.H. and Shea C.H., "The automaticity of complex motor skill learning as a function of attentional focus," Quarterly Journal of Experimental Psychologhy, vol. 54A, pp. 11431154, 2001. 
Ayse Nur AY et al./ISITES2018 Alanya - Antalya - Turkey 